\title{
ON THE DOI MODEL FOR THE SUSPENSIONS OF ROD-LIKE MOLECULES: GLOBAL-IN-TIME EXISTENCE*
}

\author{
HANTAEK BAE ${ }^{\dagger}$ AND KONSTANTINA TRIVISA
}

To Dave Levermore on his 60th birthday.

\begin{abstract}
The Doi model for the suspensions of rod-like molecules in a dilute regime describes the interaction between the orientation of rod-like polymer molecules on the microscopic scale and the macroscopic properties of the fluid in which these molecules are contained (cf. [M. Doi and S.F. Edwards, Oxford University Press, 1986]). The orientation distribution of the rods on the microscopic level is described by a Fokker-Planck-type equation on the sphere, while the fluid flow is given by the Navier-Stokes equations, which are now enhanced by an additional macroscopic stress $\sigma$ reflecting the orientation of the rods on the molecular level. Prescribing arbitrarily the initial velocity and the initial orientation distribution in suitable spaces we establish the global-in-time existence of a weak solution to our model defined on a bounded domain in three dimensional space. The proof relies on a quasi-compressible approximation of the pressure, the construction of a sequence of approximate solutions, and the establishment of compactness.
\end{abstract}

Key words. Doi model, suspensions of rod-like molecules, global-in-time existence, fluidparticle interaction model, polymeric fluids, Navier-Stokes equations, incompressible and viscous flows, Smoluchowski equation.

AMS subject classifications. 35B40, 35D05, 76N10, 35B45.

\section{Introduction}

Polymeric fluids arise in many practical applications in biotechnology, medicine, chemistry, industrial processes, and atmospheric sciences. This article deals with the Doi model for suspensions of rod-like molecules in a dilute regime. The Doi model describes the interaction between the orientation of rod-like polymer molecules at the microscopic scale and the macroscopic properties of the fluid in which these molecules are contained (cf. Doi and Edwards [10]). The macroscopic flow leads to a change of the orientation and, in the case of flexible particles, to a change in shape of the suspended microstructure. This process in turn yields the production of a fluid stress. As a first approximation, we view the identical liquid crystal molecules as inflexible rods of a thickness $b$, which is much smaller than their length $L$. In the dilute regime the rods are well separated, as expressed by $b \ll L^{-3}$. The orientation distribution of the rods $f$ is described by a Fokker-Planck-type equation,

$$
f_{t}+u \cdot \nabla_{x} f+\nabla_{\tau} \cdot\left(P_{\tau^{\perp}}\left(\nabla_{x} u \tau\right) f\right)-D_{r} \Delta_{\tau} f-D \Delta_{x} f=0,
$$

with $f$ describing the time-dependent orientation distribution that a rod with a center of mass at $x$ has an axis $\tau$ in the area element $d \tau$. Here $u=u(t, x)$ represents the velocity field, the term $u \cdot \nabla_{x} f$ characterizes the change of $f$ due to the displacement of the center of the mass of the rods by advection, whereas the drift term on the sphere $\nabla_{\tau} \cdot\left(P_{\tau^{\perp}}\left(\nabla_{x} u \tau\right) f\right)$ represents the shear-forces acting on the rods. The terms $D \Delta_{x} f, D_{r} \Delta_{\tau} f$ describe the Brownian effects: translational diffusion and rotational

\footnotetext{
* Received: December 18, 2010; accepted (in revised form): July 21, 2011. Communicated by Shi Jin.

$\dagger$ Department of Mathematics, University of California, Davis, CA 95616, U.S.A. (hantaek@math.ucdavis.edu).

$\ddagger$ Department of Mathematics \& Institute for Physical Science and Technology, University of Maryland, College Park, MD 20742-4015, U.S.A. (trivisa@math.umd.edu).
} 
diffusion respectively. $D, D_{r}$ denote the diffusivity parameters $([10,19])$. Diffusion can be seen as a gradient flow of the entropy functional

$$
E[f]:=\nu k_{B} T \int_{\Omega} \int_{S^{2}} f \ln f d \tau d x .
$$

The fluid flow is given by the Navier-Stokes equations, which are now enhanced by an additional macroscopic stress reflecting the orientation of the rods on the molecular level,

$$
(\mathrm{NS})\left\{\begin{array}{l}
u_{t}+u \cdot \nabla u-\mu \Delta u+\nabla p=\nabla \cdot \sigma \\
\nabla \cdot u=0
\end{array}\right.
$$

where $p$ denotes the pressure and $\sigma$ the macroscopic stress tensor derived from the orientation of the rods at the molecular level and is given by

$$
\sigma(t, x)=\int_{S^{2}}(3 \tau \otimes \tau-I d) f(t, x, \tau) d \tau .
$$

After normalizing $\mu, D_{r}$, and $D$ by 1 , the system of equations now reads

$$
\begin{aligned}
& f_{t}+u \cdot \nabla_{x} f+\nabla_{\tau} \cdot\left(P_{\tau^{\perp}}\left(\nabla_{x} u \tau\right) f\right)-\Delta_{\tau} f-\Delta f=0 \text { in }(0, T) \times \Omega \times S^{2}, \\
& \sigma=\int_{S^{2}}(3 \tau \otimes \tau-I d) f d \tau \quad \text { in } \quad(0, T) \times \Omega, \\
& u_{t}+u \cdot \nabla u-\Delta u+\nabla p=\nabla \cdot \sigma \quad \text { in } \quad(0, T) \times \Omega, \\
& \nabla \cdot u=0 \quad \text { in }(0, T) \times \Omega, \\
& f(0, x, \tau)=f_{0}(x, \tau) \quad \text { in } \Omega \times S^{2}, \quad u(0, x)=u_{0}(x) \text { in } \Omega,
\end{aligned}
$$

where

$$
P_{\tau^{\perp}}\left(\nabla_{x} u \tau\right)=\nabla_{x} u \tau-\left(\tau \cdot \nabla_{x} u \tau\right) \tau
$$

is the projection of $\nabla u \tau$ on the tangent space of the $(d-1)$-dimensional space $S^{d-1}$ at $\tau \in S^{d-1}$. With $\nabla_{\tau}$ and $\Delta_{\tau}$ we denote the gradient and the Laplace operator on the unit sphere, while $\nabla$ represents the gradient in $\mathbb{R}^{3}$ and

$$
(\operatorname{div} \sigma)_{i}=(\nabla \cdot \sigma)_{i}=\sum_{j=1}^{3} \frac{\partial \sigma_{i j}}{\partial x_{j}}
$$

represents the forces due to the presence of microscopic insertions.

In this paper, we consider the problem in a bounded, open, and connected domain $\Omega \subset R^{3}$ in $x$ variables. We assume that the boundary is impermeable, and that the fluid does not completely adhere to the boundary, but rather exhibits a partial slip boundary condition. That is,

$$
u \cdot \hat{n}=0, \quad u_{\text {tan }}+(D(u) \hat{n})_{\tan }=0, \quad \text { on } \partial \Omega,
$$

where tan means the tangential component a vector field at the boundary, $\hat{n}$ is the outer normal vector at the boundary, and $D(u)$ is the symmetric part of the matrix $\nabla u$. The second condition in (1.2) is called Navier's slip boundary condition, which allows that all the integrals in Definition 2.5 are finite. 
The boundary condition of $f$ in the $x$ variables is the Dirichlet boundary condition,

$$
f(t, x, \tau)=0 \quad \text { on } \quad \partial \Omega \quad \text { for almost all }(t, \tau) \in(0, T) \times S^{2},
$$

which implies the stress tensor $\sigma$ also satisfies the Dirichlet boundary condition,

$$
\sigma=0 \quad \text { on } \quad \partial \Omega .
$$

Prescribing arbitrarily the initial velocity and the initial orientation distribution in suitable spaces, we establish long-time and large data existence of a weak solution. Since the definition of a weak solution and the main result are rather complicated, we will state them in Section 2 (Definition 2.5 and Theorem 2.9).

Related results on the Doi Model for the suspensions of rod-like molecules are presented by Otto and Tzavaras in [19], where the existence of strong solutions was established in the case of perturbations of stationary homogeneous flows. The governing equations in that context involve a Fokker-Plank equation coupled with the stationary Stokes equation. The result is obtained by establishing a novel estimate for the Smoluchowski equation. Global existence of solutions in three dimensions of a model involving a Fokker-Planck equation coupled with the stationary Stokes equation is also presented by Constantin in [6]. Both articles treat a stationary Stokes equations, which allows the control of the term $\nabla_{x} u$ in terms of $\sigma$. In (cf. Constantin et al. $[7,8])$ the global well-posedness for a Fokker-Planck equation coupled with the Navier-Stokes equations in two dimensions is established. In the heart of analysis lies the use of the Littlewood-Paley decomposition method for the estimation of the $\nabla_{x} u$ in $L^{\infty}$. The global existence of a weak solution in the whole space was established by Lions and Masmoudi in [14] using the propagation of compactness argument. Linear stability analysis for a kinetic model for the sedimentation of rod-like particles is presented in [11].

The paper is organized as follows. In Section 2, we introduce the notion of a weak solution of the system (1.1) and function spaces on which weak solutions are defined. We also provide some auxiliary lemmas. In Section 3, the main result is presented. The proof relies on a quasi-compressible approximation of the pressure which is here determined as a solution of the Neumann problem, namely $p=-\frac{1}{\epsilon} \Delta^{-1}(\nabla \cdot u)$, and the mollification of the velocity field in the advection term by a suitable divergencefree mollifier. Using this approach, we can construct an approximate sequence of $\left\{u^{(\epsilon, \eta)}, p^{(\epsilon, \eta)}\right\}$, which are bounded uniformly in energy spaces, yielding the existence of a weak solution $(u, p)$. For the establishment of uniform bounds for the pressure we employ the multipliers technique of Lions [12], which involves identifying appropriate test functions in the weak formulation of the Navier-Stokes equation as solutions of a suitable Neumann problem. For the approximation of $f$ we introduce a smoothing operator $R$ in the spirit of [6] and we obtain uniform bounds of the sequence $\left\{R f^{(\epsilon, \eta)}\right\}$ in $L^{\infty}\left(0, T ; W^{1,2}(\Omega) L^{2}\left(S^{2}\right)\right) \cap L^{2}\left(0, T ; L^{2}(\Omega) W^{1,2}\left(S^{2}\right)\right)$, from which we can gain enough integrability to pass to the limit to the approximation sequence. Starting from a linear model of a Fokker-Planck equation, the analysis presented in Section 3 can accommodate more general models in the spirit of Constantin [6]. An existence result for a more general model following a line of argument similar to that in the proof of Theorem 2.9 is presented in Section 4. In Section 5 some future directions and concluding remarks are discussed.

Notation:

- $A \lesssim B$ means there is a constant $C$ such that $A \leq C B$. 
- $C(T)$ is a function in-time which only depends on the norms of initial data and $T$. - $L^{p}(0, T ; X)$ denotes the Banach set of Bochner measurable functions $f$ from $(0, T)$ to $X$ endowed with either the norm $\left(\int_{0}^{T}\|g(\cdot, t)\|_{X}^{p} d t\right)^{\frac{1}{p}}$ for $1 \leq p<\infty$ or $\sup _{t>\infty}\|g(\cdot, t)\|_{X}$ for $p=\infty$. In particular, for the density function $f, f \in L^{p}(0, T ; X Y)$ denotes $\left(\int_{0}^{T}\left\|\left(\|f(t)\|_{Y_{\tau}}\right)\right\|_{X}^{p} d t\right)^{\frac{1}{p}}$ or $\sup _{t>\infty}\left\|\left(\|f(t)\|_{Y_{\tau}}\right)\right\|_{X}$ for $p=\infty$.

- We write $(u, v)_{\Omega}$ and $<u, v>_{\Omega}$, instead of $\int_{\Omega} u v d x$ and the dual bracket, respectively. We use the same notation for the set $\Omega \times S^{2}$.

- $Q_{T}=(0, T) \times \Omega, \quad K=\Omega \times S^{2}$.

$\bullet \rightarrow, \stackrel{\star}{\rightarrow}, \rightarrow$ denote weak limit, weak star limit, and strong limit, respectively.

\section{Preliminaries}

2.1. Function spaces, Helmholtz decomposition. Let $\Omega$ be a bounded domain with a Lipschitz boundary $\partial \Omega$. The Navier boundary condition requires us to define a function space with zero normal components on the boundary. Therefore, we choose a subspace of $W^{1, r}(\Omega)$ as follows:

$$
W_{n}^{1, r}(\Omega)=\left\{v ; v \in W^{1, r}(\Omega), \quad(\operatorname{tr} v) \cdot \hat{n}=0 \quad \text { on } \quad \partial \Omega\right\},
$$

where $\operatorname{tr}$ denotes the trace operator onto the boundary. Since the velocity field is incompressible, we define a subspace of $W_{n}^{1, r}(\Omega)$ such that

$$
W_{n, d i v}^{1, r}(\Omega)=\left\{v \in W_{n}^{1, r}(\Omega) ; \nabla \cdot v=0\right\},
$$

and we define a subspace of $L^{2}(\Omega)$ with the divergence free condition:

$$
L_{n}^{2}(\Omega)={\overline{\left\{v \in W_{n, d i v}^{1,2}(\Omega)\right.}}^{\|\cdot\|_{L^{2}(\Omega)}} .
$$

We also need the notion of the dual space to define function spaces for $u_{t}$ :

$$
W_{n}^{-1, r^{\prime}}(\Omega)=\left(W_{n}^{1, r}(\Omega)\right)^{\prime}, \quad W_{n, \text { div }}^{-1, r^{\prime}}(\Omega)=\left(W_{n, \text { div }}^{1, r}(\Omega)\right)^{\prime},
$$

where $r^{\prime}$ is the conjugate of $r$.

Next, we recall the Helmholtz decomposition of a vector field. We will use it later when we construct approximate sequences by a quasi-compressible method. Let $v \in W_{n}^{1, q}(\Omega)$. Let $g$ be a solution of the following elliptic problem:

$$
\Delta g=\nabla \cdot v \quad \text { in } \quad \Omega, \quad \nabla g \cdot \hat{n}=0 \quad \text { on } \quad \partial \Omega, \quad \int_{\Omega} g d x=0 .
$$

Then, we can define the divergence-free part of $v$ as

$$
v_{d i v}=v-\nabla g .
$$

By the elliptic regularity theory,

$$
\begin{aligned}
\|g\|_{W^{2, q}(\Omega)} & \lesssim\|\nabla \cdot v\|_{L^{q}(\Omega)}, \quad\left\|v_{d i v}\right\|_{W^{1, q}(\Omega)} \lesssim\|v\|_{W^{1, q}(\Omega)}, \\
\|g\|_{W^{1, q}(\Omega)} & \lesssim\|v\|_{L^{q}(\Omega)}, \quad\left\|v_{d i v}\right\|_{L^{q}(\Omega)} \lesssim\|v\|_{L^{q}(\Omega)} .
\end{aligned}
$$


2.2. Auxiliary lemmas. When we perform the integration by parts to obtain uniform energy bounds for the velocity field $u$, we only have the $L^{2}$ norm of the symmetric part $D(u)$ of $\nabla u$, not that of $\nabla u$. To control the full derivative $\nabla u$ in terms of its symmetric part $D(u)$, we need the following lemma.

Lemma 2.1 (Korn's inequality). Let $\Omega$ be a bounded domain in $C^{1,1}$ and $1<q<\infty$. Then, for all $v \in W^{1, q}(\Omega)$ with $\operatorname{tr} v \in L^{2}(\partial \Omega)$,

$$
\|v\|_{W^{1, q}(\Omega)} \lesssim\|D(v)\|_{L^{q}(\Omega)}+\|\operatorname{tr} v\|_{L^{2}(\partial \Omega)} .
$$

Proof. For the proof of this lemma we refer the reader to Lemma 1.3 in [5].

The next lemma provides compactness of traces for relevant Bochner spaces.

Lemma 2.2. Let $q_{1} \geq 1$ and $r, q_{2} \in(1, \infty)$. Let $\mathcal{S}$ be defined as

$$
\mathcal{S}=\left\{v \in L^{\infty}\left(0, T ; L^{2}(\Omega)\right) \cap L^{r}\left(0, T ; W_{n}^{1, r}(\Omega)\right), \quad v_{t} \in L^{q_{1}}\left(0, T: W_{n, d i v}^{-1, q_{2}}(\Omega)\right)\right\} .
$$

If $\left\{v^{i}\right\}$ is bounded in $\mathcal{S}$ and $r \in\left(\frac{2 d}{d+2}, 2\right)$, then $\left\{t r v^{i}\right\}$ is precompact in $L^{p}(0, T$ : $\left.L^{s}(\partial \Omega)\right)$, where

$$
s \in\left(\frac{2 d-2}{d}, \frac{r(d-1)}{d-r}\right), \quad p<s \frac{d r+2 r-2 d}{s d-2 d+2} .
$$

Proof. See Lemma 1.4 in [5] where even more general cases are treated.

From this lemma, we can verify the following.

Lemma 2.3. Let $\left\{v^{i}\right\}$ be bounded in $\mathcal{S}$ with $d=3$ and $r=2$. Then $\left\{t r v^{i}\right\}$ is precompact in $L^{2}\left(0, T ; L^{2}(\partial \Omega)\right)$ and $L^{q}\left(0, T ; L^{\frac{4}{3}}(\partial \Omega)\right)$ for all $q \in[1, \infty)$.

The following interpolation inequality will be of use for the estimation of solutions in terms of uniform quantities which are derived from the energy estimates:

$$
\|v\|_{L^{q}(\Omega)} \lesssim\|v\|_{L^{2}(\Omega)}^{\frac{6-q}{2 q}}\|v\|_{L^{6}(\Omega)}^{\frac{3 q-6}{2 q}} \lesssim\|v\|_{L^{2}(\Omega)}^{\frac{6-q}{2 q}}\|v\|_{W^{1,2}(\Omega)}^{\frac{3 q-6}{2 q}}, \quad 2 \leq q \leq 6 .
$$

Finally, we need three simple but necessary properties of the operator $R=(1-$ $\left.\Delta_{\tau}\right)^{-\frac{s}{2}}$. Roughly speaking, the operator $R$ does not affect the advection term in the Fokker-Plank equation and it regularizes $f$ in the $\tau$ variable so that we can deal with the $L^{p}$ norm of the shear forces $\nabla_{\tau^{\prime}} \cdot\left(P_{\tau^{\perp}}\left(\nabla_{x} u \tau\right) f\right)$ in terms of the total mass of $f$.

Lemma 2.4. The operator $R=\left(1-\Delta_{\tau}\right)^{-\frac{s}{2}}, s>\frac{5}{2}$, satisfies the following properties:

$$
\begin{aligned}
& {\left[R, \nabla_{x}\right]=0} \\
& R \nabla_{\tau}: L^{1}\left(S^{2}\right) \rightarrow L^{p}\left(S^{2}\right) \quad \text { bounded for any } p>1 . \\
& R: L^{2}\left(S^{2}\right) \rightarrow H^{s}\left(S^{2}\right) \text { is bounded. }
\end{aligned}
$$

Proof. For details of the proof of this lemma, we refer the reader to [6]. 


\subsection{Main result.}

2.3.1. Definition of weak solutions. First, we introduce the assumptions on the initial data:

$$
\begin{aligned}
& u_{0} \in L^{2}(\Omega), \quad \nabla \cdot u_{0}=0, \quad R f_{0} \in L^{2}(K), \quad R=\left(1-\Delta_{\tau}\right)^{-\frac{s}{2}}, s>\frac{5}{2}, \\
& \rho_{0}(x)=\int_{S^{2}} f_{0}(x, \tau) d \tau \in L^{\infty}(\Omega) .
\end{aligned}
$$

Now, we define the notion of weak solution to (1.1).

Definition 2.5. Let $\Omega$ be a bounded domain in $C^{1,1}$. Assume that $u_{0}$ and $f_{0}$ satisfy (2.5). We say that $(u, p, f)$ is a weak solution to (1.1), with the boundary conditions (1.2), (1.3), and (1.4) if

$$
\begin{aligned}
& u \in C\left([0, T] ; L_{\text {weak }}^{2}(\Omega)\right) \cap L^{2}\left(0, T ; W_{n, d i v}^{1,2}(\Omega)\right), \quad v_{t} \in L^{\frac{5}{3}}\left(0, T ; W_{n}^{-1, \frac{5}{3}}(\Omega)\right), \\
& p \in L^{\frac{5}{3}}\left(0, T ; L^{\frac{5}{3}}(\Omega)\right), \\
& R f \in L^{\infty}\left(0, T ; L^{2}(K)\right) \cap L^{2}\left(0, T ; W^{1,2}\left(\Omega \times S^{2}\right)\right),
\end{aligned}
$$

and the following integral relations hold:

$$
\begin{aligned}
& \int_{0}^{T}\left[\left\langle u_{t}, \psi\right\rangle_{\Omega}-(u \otimes u, \nabla \psi)_{\Omega}+(D(u), D(\psi))_{\Omega}+(u, \psi)_{\partial \Omega}\right] d t \\
&= \int_{0}^{T}\left[(p, \nabla \cdot \psi)_{\Omega}+\langle\nabla \cdot \sigma, \psi\rangle_{\Omega}\right] d t \quad \text { for all } \psi \in L^{2}\left(0, T ; W_{n}^{1,2}(\Omega)\right), \\
& \int_{0}^{T}\left[\left\langle R f_{t}, \Psi\right\rangle_{K}-(R(f) u, \nabla \Psi)_{K}-\left(R\left(P_{\tau} \perp \nabla u \tau f\right), \nabla_{\tau} \Psi\right)_{K}\right. \\
&\left.+\left(R \nabla_{\tau} f, \nabla_{\tau} \Psi\right)_{K}+(R \nabla f, \nabla \Psi)_{K}\right] d t=0,
\end{aligned}
$$

for all $\Psi \in L^{\infty}\left(0, T ; W^{1,2}\left(\Omega \times S^{2}\right)\right)$.

Before formulating the main results, we provide several remarks related to the above definition.

REMARK 2.6. As one can see from the definition of weak solution of the density term $f$ in (2.8), we apply the operator $R$ on the equation of $f$ and deal with $R f$ instead of $f$. Especially, the nonlinear term $\left(R\left(P_{\tau^{\perp}} \nabla u \tau f\right), \nabla_{\tau} \Psi\right)_{K}$ can be expressed by

$$
\begin{aligned}
& \int_{\Omega} \int_{S^{2}}\left[R\left(\partial_{i} u_{j} \tau_{j} f\right) \partial_{\tau_{i}} \Psi\right] d \tau d x-\int_{\Omega} \int_{S^{2}}\left[R\left(\tau_{i} \partial_{i} u_{j} \tau_{j} f\right) \tau \cdot \nabla_{\tau} \Psi\right] d \tau d x \\
= & \int_{\Omega} \partial_{i} u_{j} \int_{S^{2}}\left(\tau_{j} f R\left(\partial_{\tau_{i}} \Psi\right)\right) d \tau d x-\int_{\Omega} \partial_{i} u_{j} \int_{S^{2}}\left(\tau_{i} \tau_{j} f R\left(\tau \cdot \nabla_{\tau} \Psi\right)\right) d \tau d x \\
= & \int_{\Omega} \partial_{i} u_{j} \int_{S^{2}}\left(R f R^{-1}\left(\tau_{j} R\left(\partial_{\tau_{i}} \Psi\right)\right)\right) d \tau d x \\
& -\int_{\Omega} \partial_{i} u_{j} \int_{S^{2}}\left(R f R^{-1}\left(\tau_{i} \tau_{j} R\left(\tau \cdot \nabla_{\tau} \Psi\right)\right)\right) d \tau d x
\end{aligned}
$$


In the sequel, we must take the limit of $\left\{R f^{(m)}\right\}$ and not $\left\{f^{(m)}\right\}$. In Section 3, we will obtain uniform bounds of $\left\{R f^{(m)}\right\}$ in $L^{\infty}\left(0, T ; L^{2}(K)\right) \cap L^{2}\left(0, T ; W^{1,2}\left(\Omega \times S^{2}\right)\right)$, which is enough to pass to the limit in the nonlinear term $\left(R\left(P_{\tau^{\perp}} \nabla u \tau f\right), \nabla_{\tau} \Psi\right)_{K}$.

REMARK 2.7. We can split (2.7) into two separate equations of $u$ and $p$. First, we take a test function $\psi$ such that $\nabla \cdot \psi=0$. Then

$$
\begin{aligned}
& \int_{0}^{T}\left[\left\langle u_{t}, \psi\right\rangle_{\Omega}-(u \otimes u, \nabla \psi)_{\Omega}+(D(u), D(\psi))_{\Omega}+(u, \psi)_{\partial \Omega}\right] d t \\
= & \int_{0}^{T}\left[\langle\nabla \cdot \sigma, \psi\rangle_{\Omega}\right] d t \text { for all } \psi \in L^{2}\left(0, T ; W_{n, \text { div }}^{1,2}(\Omega)\right) .
\end{aligned}
$$

Next, we insert $\psi=\nabla \zeta$ into (2.7), where

$$
\Delta \zeta=h, \quad \nabla \zeta \cdot n=0, \quad \int_{\Omega} \zeta=0 .
$$

Then we obtain the equation of the pressure, namely

$$
\begin{aligned}
(p, h)_{\Omega}= & \left(D(u)-u \otimes u, \nabla^{2}\left(\Delta^{-1} h\right)\right)_{\Omega}+\alpha\left(u, \nabla\left(\Delta^{-1} h\right)\right)_{\partial \Omega}-\left\langle\nabla \cdot \sigma, \nabla\left(\Delta^{-1} h\right)\right\rangle_{\Omega} \\
& \text { for a.e. } t \in(0, T), \quad \text { all } h \in L^{\infty}(\Omega), \quad \int_{\Omega} h=0 .
\end{aligned}
$$

REMARK 2.8. By the Navier boundary condition, all integrals in the definition of weak solution are finite. Moreover, Lemma 2.3 together with (2.6a) yields

$$
\operatorname{tru} \in L^{2}\left(0, T ; L^{2}(\partial \Omega)\right),
$$

so that all boundary integrals make sense.

Now, we state the main theorem of the paper.

TheOREm 2.9. Let $\Omega$ be a three dimensional bounded domain in $C^{1,1}$. Assume that $u_{0}$ and $f_{0}$ satisfy (2.5). Then, there is a weak solution $(u, p, f)$ to (1.1), with the boundary conditions (1.2), (1.3), and (1.4), satisfying all conditions in Definition 2.5.

REMARK 2.10. A smooth solution of (1.1) satisfies the free energy dissipation:

$$
\begin{aligned}
& \frac{d}{d t}\left[\|u\|_{L^{2}(\Omega)}^{2}+\int_{K}(f \log f-f+1) d \tau d x\right] \\
& \quad+4 \int_{K}\left|\nabla_{\tau} \sqrt{f}\right|^{2} d \tau d x+4 \int_{K}|\nabla \sqrt{f}|^{2} d \tau d x+\|D(u)\|_{L^{2}(\Omega)}^{2}+\alpha\|u\|_{L^{2}(\partial \Omega)}^{2}=0 .
\end{aligned}
$$

By the lower semi-continuities of functionals in the integrands, we can prove the entropy inequality under suitable conditions on the initial data. 


\section{Proof of Theorem 2.9}

3.1. Definition of $(\epsilon, \eta)$ approximations and their solutions. In the heart of the analysis lies the quasi-compressible approximation of the incompressibility condition $\nabla \cdot u=0$. Namely,

$$
\epsilon \Delta p=\nabla \cdot u \quad \text { in } \quad \Omega, \quad \nabla p \cdot n=0 \quad \text { on } \quad \partial \Omega, \quad \int_{\Omega} p d x=0 .
$$

This approximation yields the increase of the regularity of the pressure. The second key ingredient of our approach is the regularization of the convective velocity field as follows:

$$
u_{\eta}=\left(\left(\lambda_{\eta} u\right) \star w_{\eta}\right)_{d i v},
$$

where $\star w_{\eta}$ denotes the standard mollification with kernel $w$, and div means the divergent part of a vector field. Here, $\lambda_{\eta}$ is a cut-off function such that

$$
\lambda_{\eta}(x)= \begin{cases}0, & \text { if } \operatorname{dist}(x, \partial \Omega) \leq 2 \eta \\ 1, & \text { elsewhere }\end{cases}
$$

Note that if $u^{n} \rightarrow u$ in $L^{q}(\Omega \times(0, T))$ and $\nabla \cdot u=0$, then $u_{\eta}^{n} \rightarrow u$ in $L^{q}(\Omega \times(0, T))$. Now, we consider the following regularized system of equations. For simplicity of notation, we will not specify the $(\epsilon, \eta)$ dependence of functions.

$$
\begin{aligned}
& f_{t}+u_{\eta} \cdot \nabla_{x} f+\nabla_{\tau} \cdot\left(P_{\tau^{\perp}}\left(\nabla_{x} u \tau\right) f\right)-\Delta_{\tau} f-\Delta f=0 \quad \text { in } \quad(0, T) \times \Omega \times S^{2}, \\
& \sigma=\int_{S^{2}}(3 \tau \otimes \tau-I d) f d \tau \quad \text { in } \quad(0, T) \times \Omega, \\
& u_{t}+u_{\eta} \cdot \nabla u-\Delta u+\nabla p=\nabla \cdot \sigma \quad \text { in } \quad(0, T) \times \Omega, \\
& \epsilon \Delta p=\nabla \cdot u \quad \text { in } \quad(0, T) \times \Omega, \\
& f(0, x, \tau)=f_{0}(x, \tau) \quad \text { in } \quad \Omega \times S^{2}, \quad u(0, x)=u_{0}(x) \text { in } \Omega .
\end{aligned}
$$

Definition 3.1. Let $\Omega$ be a bounded domain in $C^{1,1}$. Assume that $u_{0}$ and $f_{0}$ satisfy (2.5). We say that a triple $\left(u^{(\epsilon, \eta)}, p^{(\epsilon, \eta)}, f^{(\epsilon, \eta)}\right)=(u, p, f)$ is a weak solution to (3.2), with the boundary conditions (1.2), (1.3), and (1.4) if

$$
\begin{aligned}
& u \in C\left([0, T] ; L_{\text {weak }}^{2}(\Omega)\right) \cap L^{2}\left(0, T ; W_{n, \text { div }}^{1,2}(\Omega)\right), \quad v_{t} \in L^{2}\left(0, T ; W_{n}^{-1,2}(\Omega)\right), \\
& p \in L^{2}\left(0, T ; W^{1,2}(\Omega)\right), \\
& R f \in L^{\infty}\left(0, T ; L^{2}(K)\right) \cap L^{2}\left(0, T ; W^{1,2}\left(\Omega \times S^{2}\right)\right),
\end{aligned}
$$

and the following integral relations hold:

$$
\begin{aligned}
-\epsilon(\nabla p, \nabla \pi)_{\Omega}=(\pi, \nabla \cdot u)_{\Omega} \text { for all } \pi \in W^{1,2}(\Omega) \quad \text { for a.e. } \quad t \in[0, T] \\
\\
\int_{0}^{T}\left[\left\langle u_{t}, \psi\right\rangle_{\Omega}-\left(u_{\eta} \otimes u, \nabla \psi\right)_{\Omega}+(D(u), D(\psi))_{\Omega}+(u, \psi)_{\partial \Omega}\right] d t \\
=\int_{0}^{T}\left[(p, \nabla \cdot \psi)_{\Omega}+\langle\nabla \cdot \sigma, \psi\rangle_{\Omega}\right] d t \quad \text { for all } \quad \psi \in L^{2}\left(0, T ; W_{n}^{1,2}(\Omega)\right),
\end{aligned}
$$




$$
\begin{aligned}
\int_{0}^{T}\left[\left\langle R f_{t}, \Psi\right\rangle_{K}-\left(R(f) u_{\eta}, \nabla \Psi\right)_{K}-\left(R\left(P_{\tau^{\perp}} \nabla u \tau f\right), \nabla_{\tau} \Psi\right)_{K}\right. \\
\left.+\left(R \nabla_{\tau} f, \nabla_{\tau} \Psi\right)_{K}+(R \nabla f, \nabla \Psi)_{K}\right] d t=0
\end{aligned}
$$

for all $\Psi \in L^{\infty}\left(0, T ; W^{1,2}\left(\Omega \times S^{2}\right)\right)$.

LEMMA 3.2. There exists a weak solution to the system (3.2).

Proof. The existence of a solution to the $(\epsilon, \eta)$ approximation will be established using a Galerkin approximation method. We present here the main steps of the approach and we refer the reader to [4] where similar line of argument was used in the context of elasticity.

Step 1. First, we define a linear mapping $\mathcal{F}$ that assigns to any $u \in W_{n}^{1,2}(\Omega)$ the solution $p \in W^{2,2}(\Omega)$ of the problem (3.1). Taking into consideration that $\Omega \in C^{1,1}$, the regularity theory for the Neumann problem (3.1) yields that the mapping $\mathcal{F}$ : $W_{n}^{1,2}(\Omega) \rightarrow W^{2,2}(\Omega)$ is continuous.

Step 2. Let $\left\{w_{j}\right\}_{j=1}^{\infty}$ be a orthonormal basis of $W_{n}^{1,2}(\Omega)$ that is orthonormal in $L^{2}(\Omega)$. For the construction of this basis we refer the reader to [15]. We construct the Galerkin approximations $\left\{u^{(N)}, p^{(N)}\right\}_{N=1}^{\infty}$ of the form

$$
u^{(N)}:=\sum_{i=1}^{N} d_{i}^{N}(t) w_{i}, \quad p^{(N)}:=\mathcal{F}\left(u^{(N)}\right)
$$

where $\mathbf{d}^{(\mathbf{N})}=\left(d_{1}^{N}, \ldots, d_{N}^{N}\right)$ solves the system of ordinary differential equations:

$$
\begin{aligned}
\frac{d}{d t}\left(u^{(N)}, w_{j}\right)-\left(u_{\eta}{ }^{(N)}\right. & \left.\otimes u^{(N)}, \nabla w_{j}\right) \\
+\left(D\left(u^{(N)}\right), \nabla w_{j}\right)+ & \left(u^{(N)}, w_{j}\right)_{\partial \Omega} \\
& -\left(\mathcal{F}\left(u^{(N)}\right), \nabla \cdot w_{j}\right)=\left(\nabla \cdot \sigma^{(N)}, w_{j}\right), j=1,2, \ldots, N
\end{aligned}
$$

Here, $\sigma^{(N)}=\int_{S^{2}}(3 \tau \otimes \tau-I d) f^{(N)}(t, x, \tau) d \tau$, and $f^{(N)}$ will be defined in Step 3. We require that $u^{(N)}$ satisfy the initial condition $u^{(N)}(\cdot, 0)=u_{0}^{(N)}=\sum_{j=1}^{N} d_{0}^{N} w_{j}$.

Step 3. We proceed by constructing the Galerkin approximations $\left\{f^{(N)}\right\}_{N=1}^{\infty}$. Let $\left\{y_{j}\right\}_{j=1}^{\infty}$ be an orthonormal basis of $W_{0}^{1,2}(\Omega)$ that is orthonormal in $L^{2}(\Omega)$. We need the zero boundary condition on the basis because $\sigma$ vanishes at the boundary. Let $\left\{v_{j}\right\}_{j=1}^{\infty}$ be an orthonormal basis of $W_{n}^{1,2}\left(S^{2}\right)$ that is orthonormal in $L^{2}\left(S^{2}\right)$. We construct the Galerkin approximations $\left\{R f^{(N)}\right\}_{N=1}^{\infty}$ of the form

$$
R f^{(N)}(t, \tau, x):=\sum_{i, j=1}^{N} c_{i j}^{N}(t) v_{i}(\tau) y_{j}(x)
$$

where $\mathbf{c}^{(\mathbf{N})}=\left(c_{N}^{11}, \ldots, c_{N 1}^{N}, c_{N 2}^{N}, \ldots c_{N N}^{N}\right)$ solves the following system of ordinary differ- 
ential equations:

$$
\begin{aligned}
& \frac{d}{d t}\left(R f^{(N)}, v_{i} y_{j}\right)-\left(u_{\eta}^{(N)} \cdot \nabla R f^{(N)}, v_{i} y_{j}\right) \\
& \quad+\left(R\left(P_{\tau^{\perp}} \nabla u^{(N)} \tau f^{(N)}\right), \nabla_{\tau}\left(v_{i} y_{j}\right)\right) \\
& \quad+\left(R \nabla_{\tau} f^{(N)}, \nabla_{\tau}\left(v_{i} y_{j}\right)\right)+\left(R \nabla f^{(N)}, \nabla\left(v_{i} y_{j}\right)\right)=0, \quad j=1,2, \ldots, N .
\end{aligned}
$$

We require that $R f^{(N)}$ satisfy the initial condition $R f^{(N)}(\cdot, 0)=R f_{0}^{(N)}=\sum_{i, j=1}^{N} c_{0}^{N} v_{i} y_{j}$.

Step 4. Next, we obtain uniform estimates on $\left\{u^{(N)}\right\}$. Multiplying equation (3.7) by $d_{j}^{N}$, summing over $j=1, \ldots, N$, integrating over $(0, T)$, and using the the identity

$$
\left(u_{\eta}^{(N)} \otimes u^{(N)}, \nabla u^{(N)}\right)_{\Omega}=\left(u_{\eta}^{(N)}, \nabla \frac{\left|u^{(N)}\right|^{2}}{2}\right)_{\Omega}=-\left(\nabla \cdot u_{\eta}^{(N)}, \frac{\left|u^{(N)}\right|^{2}}{2}\right)_{\Omega}=0,
$$

we get

$$
\begin{aligned}
& \frac{1}{2}\left\|u^{(N)}(t)\right\|_{L^{2}(\Omega)}^{2}+\int_{0}^{T}\left[\left\|D\left(u^{(N)}\right)\right\|_{L^{2}(\Omega)}^{2}+\left\|u^{(N)}\right\|_{L^{2}(\partial \Omega)}^{2}+\epsilon\left\|\nabla p^{(N)}\right\|_{L^{2}(\Omega)}^{2}\right] d t \\
\leq & \int_{0}^{T}\left\langle\nabla \cdot \sigma^{(N)}, u^{(N)}\right\rangle_{\Omega} d t+\left\|u_{0}^{(N)}\right\|_{L^{2}(\Omega)}^{2} \\
\leq & \int_{0}^{T}\left\|\sigma^{(N)}\right\|_{L^{2}(\Omega)}\left\|u^{(N)}\right\|_{W_{n}^{1,2}(\Omega)} d t+\left\|u_{0}\right\|_{L^{(\Omega)}}^{2}
\end{aligned}
$$

Applying Korn's inequality and Young's inequality to the last equation of (3.9) we conclude that

$$
\begin{aligned}
& \sup _{t \in(0, T)}\left\|u^{(N)}(t)\right\|_{L^{2}(\Omega)}^{2}+\int_{0}^{T}\left[\left\|u^{(N)}\right\|_{W_{n}^{1,2}(\Omega)}+\epsilon\left\|\nabla p^{(N)}\right\|_{L^{2}(\Omega)}^{2}\right] d t \\
\leq & C(T)+\int_{0}^{T}\left\|\sigma^{(N)}\right\|_{L^{2}(\Omega)} .
\end{aligned}
$$

Step 5. Next, we obtain uniform estimates on $\left\{f^{(N)}\right\}_{N=1}^{\infty}$. Multiplying equation (3.8) by $c_{i j}^{N}$, summing over $i, j=1, \ldots, N$, and integrating over $(0, T)$, we get

$$
\begin{aligned}
\sup _{t \in(0, T)}\left\|R f^{(N)}(t)\right\|_{L^{2}(K)}^{2}+\int_{0}^{T} & \int_{K}\left|\nabla_{\tau} R f^{(N)}(t)\right|^{2} d \tau d x d t \\
& +\int_{0}^{T} \int_{K}\left|\nabla R f^{(N)}(t)\right|^{2} d \tau d x d t \leq C(T),
\end{aligned}
$$

which is enough to pass to the limit in the third term of (3.6). For details of this estimation, see Section 3.3. Since

$$
\begin{aligned}
\left|\sigma^{(N)}(t, x)\right| & =\left|\int_{S^{2}}(3 \tau \otimes \tau-I d) f^{(N)}(t, x, \tau) d \tau\right| \\
& \leq \int_{S^{2}}\left|R^{-1}(3 \tau \otimes \tau-I d) R f^{(N)}(t, x, \tau)\right| d \tau \lesssim\left\|R f^{(N)}\right\|_{L^{2}\left(S^{2}\right)}
\end{aligned}
$$


we can control $\int_{0}^{T}\left\|\sigma^{(N)}\right\|_{L^{2}(\Omega)} d t$ in (3.10) by (3.11).

Step 6. To obtain compactness of the velocity we estimate the norm of its time derivative. Multiplying (3.7) by $\frac{d}{d t} c_{j}^{N}$, summing over $j=1, \ldots, N$, and integrating over time, we obtain using (3.10)

$$
\int_{0}^{T}\left(\frac{d}{d t} c^{N}\right)^{2} d t \leq C(T)
$$

By taking the limit $N \rightarrow \infty$, we show that $u^{(N)} \rightarrow u$ strongly in $C\left([0, T] ; L^{2}(\Omega)\right)$. This implies in turn that $p^{(N)} \rightarrow p$ strongly in $L^{2}\left(0, T ; W^{1,2}(\Omega)\right)$.

\subsection{Uniform estimates of $(u, p)$.}

3.2.1. Uniform estimates of $u$. Here, and in what follows, we set $(u, p, f)=$ $\left(u^{(\epsilon, \eta)}, p^{(\epsilon, \eta)}, f^{(\epsilon, \eta)}\right)$ and we derive estimates that are uniform with respect to both $\eta$ and $\epsilon$ and also estimates uniform only with respect to $\epsilon$. The existence of a weak solution to the regularized system (3.2) implies that we can take the solution as a test function to obtain uniform bounds of solutions. First, we take $\psi=u$ and $\pi=p$. Then

$$
\frac{1}{2} \frac{d}{d t}\|u\|_{L^{2}(\Omega)}^{2}+\|D(u)\|_{L^{2}(\Omega)}^{2}+\epsilon\|\nabla p\|_{L^{2}(\Omega)}^{2}+\|u\|_{L^{2}(\partial \Omega)}^{2}=\langle\nabla \cdot \sigma, u\rangle_{\Omega} .
$$

Using integration by parts in the right-hand side of (3.13), and with the aid of the boundary condition of $\sigma$, we get

$$
\frac{1}{2} \frac{d}{d t}\|u\|_{L^{2}(\Omega)}^{2}+\|D(u)\|_{L^{2}(\Omega)}^{2}+\epsilon\|\nabla p\|_{L^{2}(\Omega)}^{2}+\|u\|_{L^{2}(\partial \Omega)}^{2} \leq\|\sigma\|_{L^{2}(\Omega)}\|\nabla u\|_{L^{2}(\Omega)} .
$$

By Young's inequality and Korn's inequality,

$$
\frac{d}{d t}\|u\|_{L^{2}(\Omega)}^{2}+\|\nabla u\|_{L^{2}(\Omega)}^{2}+\epsilon\|\nabla p\|_{L^{2}(\Omega)}^{2}+\|u\|_{W_{n}^{1,2}(\Omega)}^{2} \lesssim\|\sigma\|_{L^{2}(\Omega)}^{2} .
$$

Next, we need to estimate the stress tensor $\sigma$. By (3.12) and (3.11) without $N$, we have

$$
\|\sigma\|_{L^{2}\left(Q_{T}\right)} \leq C(T)
$$

Therefore, for all $t \in[0, T]$,

$$
\|u(t)\|_{L^{2}(\Omega)}^{2}+\int_{0}^{t}\left[\|\nabla u(s)\|_{L^{2}(\Omega)}^{2}+\epsilon\|\nabla p(s)\|_{L^{2}(\Omega)}^{2}+\|u(s)\|_{W_{n}^{1,2}(\Omega)}^{2}\right] d s \leq C(T) .
$$

In particular, by the interpolation inequality (2.1), we have

$$
\int_{0}^{T}\|u(t)\|_{L^{\frac{10}{3}}}^{\frac{10}{3}} d t \leq C(T)
$$

3.2.2. Uniform estimates of $p$ and $u_{t}$. Higher integrability for the pressure $p$ is established by employing the multipliers technique of Lions [12]. This technique 
involves identifying appropriate test functions in the weak formulation of the NavierStokes equations as solutions of the following Neumann problem. In the sequel $\beta \in$ $(1,2]$.

$$
\left\{\begin{array}{l}
h=|p|^{\beta-2} p-\frac{1}{|\Omega|} \int_{\Omega}|p|^{\beta-2} p d x, \quad \text { in } \Omega \\
\nabla h \cdot n=0, \quad \text { on } \quad \partial \Omega, \quad \int_{\Omega} h d x=0 .
\end{array}\right.
$$

By the elliptic regularity theory of the Neumann problem, we have that

$$
\|\nabla h\|_{W^{1, \beta^{\prime}(\Omega)}}^{\beta^{\prime}} \lesssim\|p\|_{L^{\beta}(\Omega)}^{\beta} .
$$

Taking $\psi=\nabla h$ in (3.5) we obtain

$$
\int_{0}^{T}\|p\|_{L^{\beta}(\Omega)}^{\beta} d t=I_{1}+I_{2}+I_{3}+I_{4}+I_{5}
$$

Using the notation $Q_{T}=(0, T) \times \Omega$, and the fact $\beta \leq 2$, we get

$$
I_{1}=\int_{Q_{T}}(D(u) \cdot D(\nabla h)) d x d t \lesssim \int_{Q_{T}}|D(u)|^{\beta} d x d t+\frac{1}{8} \int_{0}^{T}\|p\|_{L^{\beta}(\Omega)}^{\beta} d t,
$$

where we use (3.19) at the first inequality. Also,

$$
\begin{gathered}
I_{2}=\int_{0}^{T}\langle\nabla \cdot \sigma, \nabla h\rangle_{\Omega} d t \lesssim \int_{0}^{T}\|\sigma\|_{L^{2}(\Omega)}^{2}+C+\frac{1}{8} \int_{0}^{T}\|p\|_{L^{\beta}(\Omega)}^{\beta} d t \\
I_{3}=\alpha \int_{0}^{T}(u, \nabla h)_{\partial \Omega} d t \lesssim \int_{0}^{T}\left[1+\|t r u\|_{L^{2}(\Omega)}^{2}\right] d t+\frac{1}{8} \int_{0}^{T}\|p\|_{L^{\beta}(\Omega)}^{\beta} d t \\
I_{4}=-\int_{Q_{T}}\left(\left(u_{\eta} \otimes u\right) \cdot \nabla^{2} h\right) d x d t \lesssim \int_{Q_{T}}\left|u_{\eta} \otimes u\right|^{\beta} d x d t+\frac{1}{8} \int_{0}^{T}\|p\|_{L^{\beta}(\Omega)}^{\beta} d t \\
I_{5}=\int_{0}^{T}\left\langle u_{t}, \nabla h\right\rangle_{\Omega} d t=-\int_{0}^{T}\left\langle(\nabla \cdot u)_{t}, h\right\rangle_{\Omega} d t \\
=-\epsilon \int_{0}^{T}\left\langle p_{t},|p|^{\beta-2} p-\frac{1}{|\Omega|} \int_{\Omega}|p|^{\beta-2} p\right\rangle_{\Omega} d t \\
=-\frac{\epsilon}{\beta}\|p(T)\|_{L^{\beta}(\Omega)}^{\beta}+\frac{\epsilon}{\beta}\|p(0)\|_{L^{\beta}(\Omega)}^{\beta}=-\frac{\epsilon}{\beta}\|p(T)\|_{L^{\beta}(\Omega)}^{\beta},
\end{gathered}
$$

where we use the fact that $p(0)=-\frac{1}{\epsilon} \nabla \cdot u_{0}=0$. Collecting all terms,

$$
\int_{0}^{T}\|p\|_{L^{\beta}(\Omega)}^{\beta} d t \lesssim C(T)+\int_{Q_{T}}\left|u_{\eta} \otimes u\right|^{\beta} d x d t
$$

Since

$$
\left\|u_{\eta} \otimes u\right\|_{L^{2}(\Omega)} \leq\left\|u_{\eta}\right\|_{L^{\infty}(\Omega)}\|u\|_{L^{2}(\Omega)} \lesssim \frac{1}{\eta}\|u\|_{L^{2}(\Omega)}
$$


the last inequality in (3.20) implies

$$
\int_{0}^{T}\|p\|_{L^{\beta}(\Omega)}^{\beta} d t \lesssim \frac{1}{\eta}
$$

If we use (3.18) instead, then

$$
\int_{0}^{T}\|p\|_{L^{\frac{5}{3}}(\Omega)}^{\frac{5}{3}} d t \leq C(T) .
$$

Finally, from the momentum equation of $u$,

$$
\left\|u_{t}\right\|_{L^{2}\left(0, T ; W_{n}^{-1,2}(\Omega)\right)} \lesssim \frac{1}{\eta}, \quad\left\|u_{t}\right\|_{L^{\frac{5}{3}}\left(0, T ; W_{n}^{-1, \frac{5}{3}}(\Omega)\right)} \lesssim C .
$$

3.3. Uniform estimates of $f$. It is clear that the function

$$
\rho(t, x)=\int_{S^{2}} f(t, x, \tau) d \tau
$$

satisfies the linear advection-diffusion equation

$$
\rho_{t}+u_{\eta} \cdot \nabla \rho-\Delta \rho=0,
$$

which implies that

$$
\rho \in L^{\infty}\left(0, T ; L^{p}(\Omega)\right), p \in[1, \infty]
$$

In particular,

$$
\|f\|_{L^{\infty}\left(0, T ; L^{\infty}(\Omega) L^{1}\left(S^{2}\right)\right)} \leq C .
$$

Next, we estimate $R f$. The existence of a weak solution to (3.2) implies that we can take $R f$ as a test function in (3.6). Let

$$
N^{2}(x, t)=\int_{S^{2}}|R f|^{2} d \tau, \quad R=\left(1-\Delta_{\tau}\right)^{-\frac{s}{2}}, \quad s>\frac{5}{2} .
$$

Then,

$$
\frac{1}{2} \frac{d}{d t} N^{2}(x, t)=\int_{S^{2}} R f \cdot R f_{t} d \tau .
$$

We calculate the right-hand side of (3.25) with the aid of the evolution equation of $f$. First, we calculate the advection term. By (2.2),

$$
-\int_{S^{2}} R f \cdot R\left(u_{\eta} \cdot \nabla f\right) d \tau=-\frac{1}{2} u_{\eta} \cdot \nabla_{x} \int_{S^{2}}|R f|^{2} d \tau .
$$

Secondly, we calculate the diffusion terms:

$$
\begin{gathered}
\int_{S^{2}} R f \cdot R\left(\Delta_{\tau} f+\Delta f\right) d \tau=-\int_{S^{2}}\left|\nabla_{\tau} R f\right|^{2} d \tau-\int_{S^{2}}|\nabla R f|^{2} d \tau \\
+\frac{1}{2} \Delta \int_{S^{2}}|R f|^{2} d \tau
\end{gathered}
$$


Finally, we calculate the drift term in $\tau$ :

$$
\begin{aligned}
\int_{S^{2}} R f \cdot R \nabla_{\tau} \cdot\left(P_{\tau^{\perp}}\left(\nabla_{x} u \tau\right) f\right) d \tau & \lesssim\|R f\|_{L^{2}\left(S^{2}\right)}\left\|R \nabla_{\tau} \cdot\left(P_{\tau^{\perp}}\left(\nabla_{x} u \tau\right) f\right)\right\|_{L^{2}\left(S^{2}\right)} \\
& \lesssim\left|\nabla_{x} u\right| \cdot\|R f\|_{L^{2}\left(S^{2}\right)}\|f\|_{L^{1}\left(S^{2}\right)} \\
& \lesssim\left|\nabla_{x} u\right| \cdot\|R f\|_{L^{2}\left(S^{2}\right)}
\end{aligned}
$$

where we use (2.3) in the second inequality and (3.24) in the last inequality. Combining all terms, we have

$$
\frac{d}{d t} N^{2}+u_{\eta} \cdot \nabla_{x} N^{2}-\Delta N^{2}+\int_{S^{2}}\left|\nabla_{\tau} R f\right|^{2} d \tau+\int_{S^{2}}|\nabla R f|^{2} d \tau \lesssim\left|\nabla_{x} u\right| N .
$$

Integrating (3.29) over $\Omega$ we arrive at

$$
\begin{aligned}
& \frac{d}{d t} \int_{\Omega} N^{2} d x+\int_{K}\left|\nabla_{\tau} R f\right|^{2} d \tau d x \int_{K}|\nabla R f|^{2} d \tau d x \\
\lesssim & \int_{\Omega}|\nabla u| N d x \leq\|\nabla u\|_{L^{2}(\Omega)}\left(\int_{\Omega} N^{2} d x\right)^{\frac{1}{2}} .
\end{aligned}
$$

Since

$$
\int_{0}^{T}\|\nabla u(t)\|_{L_{x}^{2}(\Omega)}^{2} d t \leq C(T)
$$

for all $t \in[0, T]$,

$$
\left\|N^{2}(t)\right\|_{L^{1}(\Omega)}+\int_{0}^{t} \int_{K}\left|\nabla_{\tau} R f\right|^{2} d \tau d x d s+\int_{0}^{t} \int_{K}|\nabla R f|^{2} d \tau d x d s \leq C(T),
$$

which means that $R f$ is bounded in $L^{\infty}\left(0, T ; L^{2}(K)\right) \cap L^{2}\left(0, T ; W^{1,2}\left(\Omega \times S^{2}\right)\right)$. We note that we can obtain (3.28) because $R \nabla_{\tau} \cdot\left(P_{\tau^{\perp}}\left(\nabla_{x} u \tau\right) f\right)$ is not a generic quadratic term under the operator $R$. Therefore, we can avoid taking the $L^{\infty}(\Omega)$ norm of $|\nabla u|$.

3.4. Passing to the limits. Now, we would like to take the limit of the approximate sequence which is uniformly bounded in $(\epsilon, \eta)$. First, we take the limit in $\epsilon$, and then will take the limit in $\eta$. The limiting process in $u$ follows a similar line of argument to the one presented in [4]. For the completeness, we present the details here.

3.4.1. Passing to the limit in $u$. First, we take the limit in $\epsilon$. It follows from (3.17), (3.18), (3.21), the first term in (3.23), and by the Aubin-Lions lemma there exist subsequences (not labeled, without indicating $\eta$ dependence) and $(u, p, \sigma)$ such that

$$
\begin{aligned}
& u_{t}^{\epsilon} \rightarrow u_{t} \quad \text { in } \quad L^{2}\left(0, T ; W_{n}^{-1,2}(\Omega)\right), \\
& u^{\epsilon} \rightarrow u \quad \text { in } L^{2}\left(0, T ; W_{n}^{1,2}(\Omega)\right), \\
& u^{\epsilon} u \quad \text { in } \quad L^{\infty}\left(0, T ; L^{2}(\Omega)\right), \\
& u^{\epsilon} \rightarrow u \quad \text { in } \quad L^{q}\left(0, T ; L^{q}(\Omega)\right) \quad \text { for } \quad 1 \leq q<\frac{10}{3}, \\
& \nabla u^{\epsilon} \rightarrow \nabla u \quad \text { in } \quad L^{2}\left(0, T ; L^{2}(\Omega)\right),
\end{aligned}
$$




$$
\begin{aligned}
& p^{\epsilon} \rightarrow p \quad \text { in } \quad L^{2}\left(0, T ; L^{2}(\Omega)\right), \\
& \sigma^{\epsilon} \rightarrow \sigma \quad \text { in } \quad L^{2}\left(0, T ; L^{2}(\Omega)\right) .
\end{aligned}
$$

Moreover, from Lemma 2.3,

$$
\text { tru } u^{\epsilon} \rightarrow \text { tru } \quad \text { in } \quad L^{2}\left(0, T ; L^{2}(\partial \Omega)\right) .
$$

We also observe that for $\psi \in L^{2}\left(0, T ; W^{1,2}(\Omega)\right)$,

$$
\begin{aligned}
\left|\int_{0}^{T}(\nabla \cdot u, \psi)_{\Omega} d t\right| & =\lim _{\epsilon \rightarrow 0}\left|\int_{0}^{T}\left(\nabla \cdot u^{\epsilon}, \psi\right)_{\Omega} d t\right|=\lim _{\epsilon \rightarrow 0}\left|\int_{0}^{T} \int_{\Omega}\left(\nabla \psi \cdot \nabla p^{\epsilon}\right) d x d t\right| \\
& \leq \lim _{\epsilon \rightarrow 0} \sqrt{\epsilon}\left(\int_{Q_{T}}|\nabla \psi|^{2}\right)^{\frac{1}{2}}\left(\int_{Q_{T}} \epsilon\left|\nabla p^{\epsilon}\right|^{2}\right)^{\frac{1}{2}}=0,
\end{aligned}
$$

which implies $\nabla \cdot u=0$ a.e. in $(0, T) \times \Omega$. The above convergence is enough to take the limit in (3.5), and $(u, p, \sigma)=\left(u^{\eta}, p^{\eta}, \sigma^{\eta}\right)$ satisfies

$$
\begin{aligned}
& \int_{0}^{T}\left[\left\langle u_{t}, \psi\right\rangle_{\Omega}-\left(u_{\eta} \otimes u, \nabla \psi\right)_{\Omega}+(D(u), D(\psi))_{\Omega}+(u, \psi)_{\partial \Omega}\right] d t \\
= & \int_{0}^{T}\left[(p, \nabla \cdot \psi)_{\Omega}+\langle\nabla \cdot \sigma, \psi\rangle_{\Omega}\right] d t \quad \text { for all } \psi \in L^{2}\left(0, T ; W_{n}^{1,2}(\Omega)\right) .
\end{aligned}
$$

Secondly, we take the limit in $\eta$. We know that $\left(u^{\eta}, p^{\eta}, \sigma^{\eta}\right)$ satisfies (3.5). From (3.17), (3.18), (3.22), and the second term in (3.23), there exists a subsequence and a limit $(u, p, \sigma)$ such that

$$
\begin{aligned}
& u_{t}^{\eta} \rightarrow u_{t} \quad \text { in } \quad L^{\frac{5}{3}}\left(0, T ; W_{n}^{-1, \frac{5}{3}}(\Omega)\right), \\
& u^{\eta} \rightarrow u \quad \text { in } L^{2}\left(0, T ; W_{n}^{1,2}(\Omega)\right), \\
& u^{\eta} \stackrel{\star}{\rightarrow} u \quad \text { in } \quad L^{\infty}\left(0, T ; L^{2}(\Omega)\right), \\
& u^{\eta} \rightarrow u \quad \text { in } \quad L^{q}\left(0, T ; L^{q}(\Omega)\right) \quad \text { for } \quad 1 \leq q<\frac{10}{3}, \\
& \nabla u^{\eta} \rightarrow \nabla u \quad \text { in } \quad L^{2}\left(0, T ; L^{2}(\Omega)\right), \\
& t r u^{\eta} \rightarrow t r u \quad \text { in } \quad L^{2}\left(0, T ; L^{2}(\partial \Omega)\right), \\
& p^{\eta} \rightarrow p \quad \text { in } L^{\frac{5}{3}}\left(0, T ; L^{\frac{5}{3}}(\Omega)\right), \\
& \sigma^{\epsilon} \rightarrow \sigma \quad \text { in } L^{2}\left(0, T ; L^{2}(\Omega)\right) .
\end{aligned}
$$

Therefore, we can take the limit in (3.5).

3.4.2. Passing to the limit in $f$. In order to take the limit in (3.6), we employ the compactness of $\left\{R f^{(N)}\right\}_{N=1}^{\infty}$. Since there are two indices $(\epsilon, \eta)$ involved, we need to use it twice. Here we only present one step. Let $m$ denote either $\epsilon$ or $\eta$. From the boundedness of $\left\{R f^{(m)}\right\}$ in $L^{\infty}\left(0, T ; L^{2}(K)\right) \cap L^{2}\left(0, T ; W^{1,2}\left(\Omega \times S^{2}\right)\right)$, we can extract a subsequence, $\left\{R f^{\left(m_{j}\right)}\right\}$ converging to $R f$ strongly in $L^{2}\left(0, T ; L^{2}(K)\right)$. Therefore, we can pass to the limit in (3.6), and this completes the proof of Theorem 2.9 .

REMARK 3.3. Related results have been obtained by Lions and Masmoudi in [14] where the existence of a weak solution of (1.1) with $D=0$ on the whole space 
was obtained using the propagation of compactness argument. Using an argument employing defect measures, it was deduced that

$$
\frac{d}{d t} \int_{S^{2}} \nu d \tau+u \cdot \nabla_{x} \int_{S^{2}} \nu d \tau \lesssim\left(1+|\nabla u|^{2}\right) \int_{S^{2}} \nu d \tau
$$

which yields that $\int_{S^{2}} \nu d \tau=0$ if $\int_{S^{2}} \nu_{0} d \tau=0$.

In the same spirit a related result can be obtained in the present context for $D=0$. Indeed, introducing the following defect measures:

$$
\left|R\left(f^{(m)}-f\right)\right|^{2} \rightarrow \nu, \quad\left|\nabla u^{(m)}-\nabla u\right|^{2} \rightarrow \beta, \quad\left|\sigma^{(m)}-\sigma\right|^{2} \rightarrow \alpha,
$$

and taking into consideration relations (3.12) (without $N$ ) and (3.15), we get

$$
|\sigma| \lesssim\|R f\|_{L^{2}\left(S^{2}\right)}, \quad\|\nabla u\|_{L^{2}\left(Q_{T}\right)} \lesssim\|\sigma\|_{L^{2}\left(Q_{T}\right)}
$$

from which we deduce that

$$
\beta \lesssim \alpha \lesssim \int_{S^{2}} \nu d \tau
$$

Using the propagation of compactness argument in the spirit of Lions and Masmoudi [14] one establishes that in fact $\left\{R f^{\left(m_{j}\right)}\right\}$ converges strongly to $R f$ in $L^{2}\left(0, T ; L^{2}(K)\right)$, yielding that $\int_{s^{2}} \nu d \tau=0$, which implies that $\beta=\alpha=0$. We note that $\left\{\sigma^{(m)}\right\}$ and $\left\{\nabla u^{(m)}\right\}$ converge strongly in $L^{2}\left(0, T ; L^{2}(\Omega)\right)$, and this strong convergence cannot be derived simply from the energy bounds. The propagation of compactness argument guarantees that if one is able to construct a sequence of weak solutions which converges weakly and such that the initial data converges strongly then the weak limit is also a solution. We refer the reader to the article by Lions and Masmoudi [13] where the details of this argument are given in the context of the whole space.

REMARK 3.4. Since the problem is posed on a bounded domain, the $L^{2}$ decay of $u$ and $\rho$ can be established. First, for $\rho_{0} \in L^{2}$ with $\rho=0$ on $\partial \Omega$,

$$
\frac{d}{d t}\|\rho(t)\|_{L^{2}(\Omega)}^{2} \leq-\|\nabla \rho(t)\|_{L^{2}(\Omega)}^{2} \leq-C_{1}\|\rho(t)\|_{L^{2}(\Omega)}^{2},
$$

where Poincare's inequality is used at the second inequality. Therefore,

$$
\|\rho(t)\|_{L^{2}}^{2} \lesssim e^{-C_{1} t}
$$

Since $|\sigma| \leq C \rho$, we also have

$$
\|\sigma(t)\|_{L^{2}}^{2} \lesssim e^{-C_{1} t}
$$

Notice that $u$ satisfies

$$
\frac{d}{d t}\|u(t)\|_{L^{2}(\Omega)}^{2} \leq-\|\nabla u(t)\|_{L^{2}(\Omega)}^{2}+\|\sigma\|_{L^{2}(\Omega)}^{2} \leq-C_{2}\|u(t)\|_{L^{2}(\Omega)}^{2}+e^{-C_{1} t},
$$

where we use Poincare's inequality at the second inequality. We note that the Poincare inequality still holds for the Neunmann boundary condition $u \cdot n=0$ since $u$ is divergence-free. For the details, see the appendix of [22]. Therefore,

$$
\|u(t)\|_{L^{2}}^{2} \lesssim e^{-C t}, \quad C=\min \left\{C_{1}, C_{2}\right\}
$$




\section{A more general model}

In the spirit of Constantin [6] we consider now more general models. More specifically, the system of equations is given by

$$
\begin{aligned}
& f_{t}+u \cdot \nabla f+\nabla_{\tau} \cdot\left(P_{\tau^{\perp}}\left(\nabla_{x} u \tau\right) f+\nabla_{\tau} U f\right)-\Delta_{\tau} f-\Delta f=0 \quad \text { in } \quad(0, T) \times \Omega \times S^{2}, \\
& \sigma=\int_{S^{2}}(3 \tau \otimes \tau-I d) f d \tau \quad \text { in } \quad(0, T) \times \Omega \\
& u_{t}+u \cdot \nabla u-\Delta u+\nabla p=\nabla \cdot \sigma \quad \text { in } \quad(0, T) \times \Omega \\
& \nabla \cdot u=0 \quad \text { in } \quad(0, T) \times \Omega, \\
& f(0, x, \tau)=f_{0}(x, \tau) \quad \text { in } \quad \Omega \times S^{2}, \quad u(0, x)=u_{0}(x) \quad \text { in } \quad \Omega
\end{aligned}
$$

where the potential $U$ is

$$
U(t, x, \tau)=\int_{S^{2}} Z(\tau, w) f(t, x, w) d w,
$$

with a kernel $Z$ which is a smooth, time and space independent symmetric function $Z: S^{2} \times S^{2} \rightarrow \mathbb{R}$. The macroscopic stress tensor $\sigma$ consists of two parts:

$$
\sigma=\sigma^{(1)}+\sigma^{(2)},
$$

where $\sigma^{(1)}$ is the same as $\sigma$ in $(1.1 \mathrm{~b})$, and $\sigma^{(2)}$ is

$$
\sigma_{i j}^{(2)}(t, x)=\int_{S^{2}} \int_{S^{2}} \gamma_{i j}^{(2)}(m, n) f(t, x, m) f(t, x, n) d m d n,
$$

where $\gamma_{i j}^{(2)}$ are smooth, time independent, space independent, and do not depend on $f$. Now, we define a weak solution of this system (4.1). The definition is identical to Definition 2.5 except for the two extra terms $\nabla_{\tau} U f$ and $\sigma^{(2)}$.

Definition 4.1. Let $\Omega$ be a bounded domain in $C^{1,1}$. Assume that $u_{0}$ and $f_{0}$ satisfy (2.5). We say that $(u, p, f)$ is a weak solution to (4.1), with the boundary conditions (1.2), (1.3), and (1.4), if

$$
\begin{aligned}
& u \in C\left([0, T] ; L_{\text {weak }}^{2}(\Omega)\right) \cap L^{2}\left(0, T ; W_{n, d i v}^{1,2}(\Omega)\right), \quad v_{t} \in L^{\frac{5}{3}}\left(0, T ; W_{n}^{-1, \frac{5}{3}}(\Omega)\right), \\
& p \in L^{\frac{5}{3}}\left(0, T ; L^{\frac{5}{3}}(\Omega)\right), \\
& R f \in L^{\infty}\left(0, T ; L^{2}(K)\right) \cap L^{2}\left(0, T ; W^{1,2}\left(\Omega \times S^{2}\right)\right),
\end{aligned}
$$

and the following integral relations hold:

$$
\begin{aligned}
& \int_{0}^{T}\left[\left\langle u_{t}, \psi\right\rangle_{\Omega}-(u \otimes u, \nabla \psi)_{\Omega}+(D(u), D(\psi))_{\Omega}+(u, \psi)_{\partial \Omega}\right] d t \\
&= \int_{0}^{T}\left[(p, \nabla \cdot \psi)_{\Omega}+\langle\nabla \cdot \sigma, \psi\rangle_{\Omega}\right] d t \quad \text { for all } \psi \in L^{2}\left(0, T ; W_{n}^{1,2}(\Omega)\right), \\
& \int_{0}^{T}\left[\left\langle R f_{t}, \Psi\right\rangle_{K}-(R(f) u, \nabla \Psi)_{K}-\left(R\left(P_{\tau^{\perp}} \nabla u \tau f\right), \nabla_{\tau} \Psi\right)_{K}\right. \\
&\left.\quad+\left(R\left(\nabla_{\tau} U f\right), \nabla_{\tau} \Psi\right)_{K}+\left(R \nabla_{\tau} f, \nabla_{\tau} \Psi\right)_{K}+(R \nabla f, \nabla \Psi)_{K}\right] d t=0,
\end{aligned}
$$


for all $\Psi \in L^{\infty}\left(0, T ; W^{1,2}\left(\Omega \times S^{2}\right)\right)$.

Notice the presence of the extra nonlinear terms in the system (4.1). The following result can now be proved.

TheOREM 4.2. Let $\Omega$ be a three dimensional bounded domain in $C^{1,1}$. Assume that $u_{0}$ and $f_{0}$ satisfy (2.5). Then, there is a weak solution $(u, p, f)$ to (4.1), with the boundary conditions (1.2), (1.3), and (1.4), satisfying all conditions in Definition 4.1.

Proof. The proof of Theorem 4.2 follows a similar line of argument as the proof of Theorem 2.9, hence we only provide the main outline of the proof and the estimation of the extra terms.

Step 1. We construct the $(\epsilon, \eta)$ approximating scheme which now reads

$$
\begin{aligned}
& f_{t}+u_{\eta} \cdot \nabla_{x} f+\nabla_{\tau} \cdot\left(P_{\tau^{\perp}}\left(\nabla_{x} u \tau\right) f+\nabla_{\tau} U f\right)-\Delta_{\tau} f-\Delta f=0 \quad \text { in }(0, T) \times \Omega \times S^{2}, \\
& u_{t}+u_{\eta} \cdot \nabla u-\Delta u+\nabla p=\nabla \cdot \sigma \quad \text { in }(0, T) \times \Omega, \\
& \epsilon \Delta p=\nabla \cdot u \text { in }(0, T) \times \Omega, \\
& f(0, x, \tau)=f_{0}(x, \tau) \text { in } \Omega \times S^{2}, \quad u(0, x)=u_{0}(x) \text { in } \Omega .
\end{aligned}
$$

Step 2. Next we establish the existence of a weak solution to this system following the line of argument in Section 3. In the heart of the analysis lie the establishment of uniform estimates on $\{u, p\}$. This requires a uniform estimate of the stress tensor $\sigma$. Here we focus on the quadratic component $\sigma^{(2)}$ of the stress tensor $\sigma$. Observe that

$$
\begin{aligned}
\left|\sigma_{i j}^{(2)}(t, x)\right| & \leq\left|\int_{S^{2}} f(t, x, n) \int_{S^{2}} R^{-1} \gamma_{i j}^{(2)}(m, n) R f(t, x, n) d m d n\right| \\
& \lesssim\|f\|_{L^{1}\left(S^{2}\right)}\|R f\|_{L^{2}\left(S^{2}\right)} \lesssim\|R f\|_{L^{2}\left(S^{2}\right)}
\end{aligned}
$$

Therefore both terms $\sigma^{(1)}$ and $\sigma^{(2)}$ and as a consequence the stress tensor $\sigma$ can be handled in the same fashion as (3.12).

Step 3. Next, we establish uniform estimates of $f$ following the approach presented in Section 3.3. For the sake of completeness we present here the estimate for the extra drift term in $\tau$, namely

$$
\begin{aligned}
& \int_{S^{2}} R f \cdot R \nabla_{\tau} \cdot\left(f \nabla_{\tau} U\right) d \tau \lesssim\|R f\|_{L^{2}\left(S^{2}\right)}\left\|f \nabla_{\tau} U\right\|_{L^{1}\left(S^{2}\right)} \\
& \lesssim\|R f\|_{L^{2}\left(S^{2}\right)}\|f\|_{L^{1}\left(S^{2}\right)}\left\|\nabla_{\tau} U\right\|_{L^{\infty}\left(S^{2}\right)} \lesssim\|R f\|_{L^{2}\left(S^{2}\right)}\|f\|_{L^{1}\left(S^{2}\right)}^{2} \lesssim\|R f\|_{L^{2}\left(S^{2}\right)} .
\end{aligned}
$$

The uniform estimate on $R f$ can now be established following the line of argument presented in Section 3.3.

\section{Concluding remarks}

The present article is part of a research program whose objective is the investigation of general models for polymeric fluids in domains with complex geometries. Nonlinear Fokker-Planck-type equations coupled with Navier-Stokes equations in which the added stresses $\sigma$ depend in either linearly or nonlinearly on the density of particles are of great scientific interest. The quantity $\operatorname{div} \sigma$ represents the forces due to the presence of microscopic insertions. The insertions are objects parameterized by a microscopic variable $\tau$, which belongs to a general manifold $M$ of dimension $d$. In the case of rod-like particles (as the one in the present article) the manifold $M$ is 
the unit sphere in three dimensions, and $\tau \in S^{2}$ represents the director of the rods. More complicated particles require more degrees of freedom for the space $M$. As mentioned in [6], articulated rods with several articulations require a phase space which is a product of spheres. Motivated by engineering applications which often involve manifolds which fail to be connected (domains with holes and other deformations) one of the goals of this project is the treatment of general manifolds $M$.

Among the models of great scientific interest are included the Finite Extensible Nonlinear Elastic (FENE) dumbbell model of polymeric flows for a general class of potentials and the investigation of relevant models in viscoelasticity of Oldroyd B-type or Johnson-Segalman-type $[17,18]$. In the FENE model, a polymer is idealized as an elastic dumbbell consisting of two beads joined by a spring which can be represented by a vector $R$ (cf. Bird, Curtis, Amstrong, and Hassager [2, 3], Doi and Edwards [10] for some physical introduction to the models, and Ottinger [20] for a mathematical treatment, and Owens and Phillips [21] for the computational aspects.) We refer the reader also to the recent work of Masmoudi [16] for results relevant to the existence of weak solutions to such models.

We remark that the investigation of singular limits of complex fluids for compressible flows over bounded domains is of great scientific interest, physically relevant, and presents new challenges in the analysis. The issue of existence of solutions to the Doi model for compressible flows is considered by the authors in a forthcoming article [1]. Note that unlike the cases involving the whole domain or exterior domains where acoustic waves are damped locally due to dispersive effects of the wave equation, the main obstacle in the treatment of bounded domains is the persistence of the fast waves over these domains. Therefore in general one can only expect weak convergence of the solutions. It is worth noting that there are situations where strong convergence can be achieved due to the interaction of acoustic waves with the boundary of the domain, where a thin boundary layer is created to damp the energy carried by these fast oscillations. This phenomenon has been observed for both asymptotics of fluid equations and hydrodynamic limits of kinetic equations. It is therefore natural to ask whether similar phenomena happen for models of polymeric fluids. Before attempting to answer this question, one needs to know what are the physical boundary conditions that should be imposed on such systems. These boundary conditions are typically derived from the underlying kinetic equations so that they are compatible with the given boundary conditions for the kinetic equations. Deriving admissible boundary conditions for models describing the evolution of polymeric fluids, establishing the well-posedness theory of such systems, and investigating their asymptotics over bounded domains are some of the goals of this program.

Acknowledgments. H.B. gratefully acknowledges the support provided by the Center for Scientific Computation and Mathematical Modeling (CSCAMM) at the University of Maryland where this research was performed. H.B. is partially supported by NSF grants DMS 07-07949, DMS 10-08397, and ONR grant N000140910385. K.T. acknowledges the support by the National Science Foundation under the awards DMS-0807815, DMS-0405853, and DMS-1109397. K.T. would like to thank Thanos Tzavaras who brought the Doi model to her attention. The authors are grateful to the referees whose comments improved the presentation of the article. 


\section{REFERENCES}

[1] H. Bae and K. Trivisa, On the Doi Model for the suspensions of rod-like molecules in compressible fluids, Math. Models Meth. Appl. Sci., 22(10), 1250027, 2012.

[2] R.B. Bird, R. Amstrong, and O. Hassager, Dynamics of Polymeric Liquids, Wiley, New York, $1,1977$.

[3] R.B. Bird, C. Curtiss, R. Amstrong, and O. Hassager, Dynamics of Polymeric Liquids, Kinetic Theory, Wiley, New York, 2, 1987.

[4] M. Bulicek, E. Feireisl, and J. Malek, A Navier-Stokes-Fourier system for incompressible fluids with temperature dependent material coefficients, Nonlin. Anal. Real World Appl., 10(2), 992-1015, 2009.

[5] M. Bulicek, J. Malek, and K.R. Rajagopal, Navier's slip and evolutionary Navier-Stokes like systems with pressure and shear-rate dependent viscosity, Indian Univ. Math., J., 56(1), 41-50, 2007.

[6] P. Constantin, Nonlinear Fokker-Planck Navier-Stokes systems, Commun. Math. Sci., 3(4), 531-544, 2005.

[7] P. Constantin, C. Fefferman, E.S. Titi, and A. Zarnescu, Regularity of coupled two-dimensional nonlinear Fokker-Planck and Navier-Stokes systems, Commun. Math. Phys., 270(3), 789811, 2007.

[8] P. Constantin and N. Masmoudi, Global well-posedness for a Smoluchowski equation coupled with Navier-Stokes equations in 2D, Commun. Math. Phys., 278(1), 179-191, 2008.

[9] R.J. Diperna and P.L. Lions, Ordinary differential equations, transport theory and Sobolev spaces, Invent. Math., 98(3), 511-547, 1989

[10] M. Doi and S.F. Edwards, The Theory of Polymer Dynamics, Oxford University Press, 1986.

[11] C. Helzel, F. Otto, and A. Tzavaras, A kinetic model for the sedimentation of rod-like particles, preprint, 2010.

[12] P.L. Lions, Mathematical Topics in Fluid Dynamics, Vol.2: Compressible Models, Oxford Science Publication, Oxford, 1998.

[13] P.L. Lions and N. Masmoudi, Global solutions for some Oldroyd models of non-Newtonian flows, Chinese Ann. Math. Ser. B, 21(2), 131-146, 2000.

[14] P.L. Lions and N. Masmoudi, Global solutions of weak solutions to some micro-macro models, C.R. Math. Sci. Paris, 345(1), 15-20, 2007.

[15] J. Málek, J. Necas, M. Rokyta, and M. Ruzicka, Weak and measure valued solutions to evolutionary PDEs, Chapman \& Hall, London, 1996.

[16] N. Masmoudi, Global existence of weak solutions to the FENE Dumbbell model of polymeric flows, Inv. math., 191(2), 427-500, 2013.

[17] J.A. Nohel and P.L. Pego, On the generation of discontinuous shearing motions of a nonNewtonian fluid, Arch. Rational Mech. Anal., 139, 355-376, 1997.

[18] J.A. Nohel, R.L. Pego, and A.E. Tzavaras, Stability of discontinuous steady states in shearing motions of a non-Newtonian fluid, Proc. Roy. Soc. Edinburgh, 115A, 39-59, 1990.

[19] F. Otto and A Tzavaras, Continuity of velocity gradients in suspensions of rod-like molecules, Commun. Math. Phys., 277(3), 729-758, 2008.

[20] H.C. Öttinger, Stochastic Processes in Polymeric Fluids, Tools and Examples for Developing Simulation Algorithms, Springer-Verlag, Berlin, 1996.

[21] R.G. Owens and T.N. Phillips, Computational Rheology, Imperial College Press, London, 2002.

[22] R. Temam, Navier-Stokes Equations: Theory and Numerical Analysis, AMS Chelsea Publishing, 2001. 